\title{
Condensed Phenoxazine Dimer and Its Radical Cation
}

\author{
Takayuki Miyamae, Makoto Haraguchi, Yoshimitsu Tachi,
}

Shuichi Suzuki, Masatoshi Kozaki, and Keiji Okada

\begin{tabular}{|c|c|}
\hline Citation & Organic Letters. 22(17); 6790-6793. \\
\hline Issue Date & 2020-08-19 \\
\hline Type & Journal Article \\
\hline Textversion & Author \\
\hline $\begin{array}{l}\text { Supporting } \\
\text { Information }\end{array}$ & $\begin{array}{l}\text { The Supporting Information is available free of charge at } \\
\text { https://pubs.acs.org/doi/10.1021/acs.orglett.0c02305. } \\
\text { Detailed experimental procedures, characterization data, X-ray data with packing } \\
\text { structure, copies of } 1 \mathrm{H} \text { and } 13 \mathrm{C} \text { NMR spectra of products, and DFT calculations (PDF) }\end{array}$ \\
\hline Rights & $\begin{array}{l}\text { This document is the Accepted Manuscript version of a Published Work that appeared } \\
\text { in final form in Organic Letters, copyright } @ \text { American Chemical Society after peer } \\
\text { review and technical editing by the publisher. To access the final edited and published } \\
\text { work see https://doi.org/10.1021/acs.orglett.0c02305. }\end{array}$ \\
\hline DOI & 10.1021/acs.orglett.0c02305 \\
\hline
\end{tabular}

\author{
Self-Archiving by Author(s) \\ Placed on: Osaka City University Repository
}

Miyamae, T., Haraguchi, M., Tachi, Y., Suzuki, S., Kozaki, M., \& Okada, K. (2020). Condensed Phenoxazine Dimer and Its Radical Cation. Organic Letters, 22(17), 6790-6793.

https://doi.org/10.1021/acs.orglett.0c02305 


\title{
Condensed Phenoxazine Dimer and Its Radical Cation
}

\author{
Takayuki Miyamae, ${ }^{\dagger}$ Makoto Haraguchi, ${ }^{\dagger}$ Yoshimitsu Tachi, ${ }^{\dagger}$ Shuichi Suzuki, ${ }^{\S}, *$ Masatoshi \\ Kozaki $^{\dagger, *, *}$ and Keiji Okada ${ }^{\dagger, \ddagger}$ \\ ${ }^{\dagger}$ Graduate School of Science, Osaka City University, Sugimoto, Sumiyoshi-ku, Osaka 558-8585 (Japan) \\ Osaka City University Advanced Research Institute for Natural Science and Technology (OCARINA) Sugimoto, \\ Sumiyoshi-ku, Osaka 558-8585 (Japan) \\ ${ }^{\S}$ Graduate School of Engineering Science, Osaka University, Toyonaka, Osaka 560-8531 (Japan) \\ Supporting Information Placeholder
}

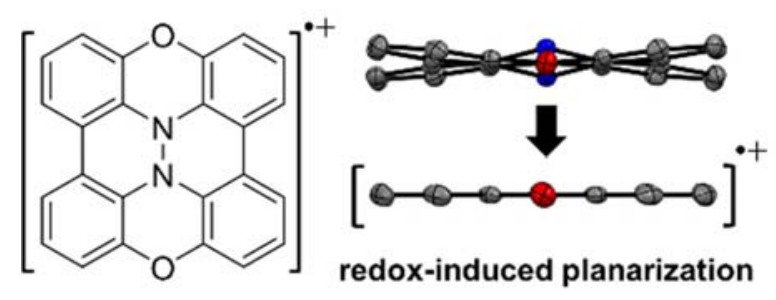

\begin{abstract}
Condensed phenoxazine dimer was synthesized and characterized. X-ray crystallographic analysis of the dimer shows a double-butterfly structure, in which the nitrogen atoms are located above and below the molecular plane. Radical cation salt of the dimer was obtained using tris(4-bromophenyl)aminium hexafluoroantimonate as the oxidant. The salt is air-stable in solid and solution states. The cation structure was evaluated by X-ray crystallographic analysis, showing that the phenoxazine units were converted to a planar structure upon oxidation.
\end{abstract}

Radical cations of condensed polycyclic aromatic compounds are an important class of materials in the development of organic electronic, optical, and magnetic devices. ${ }^{1}$ Phenothiazine PTZ and phenoxazine PXZ produce stable radical cations upon one-electron oxidation, converting the unique butterfly structure into planar. ${ }^{2}$ The structural transformation is followed by a dramatic change in the photophysical properties and $\pi$-electron delocalization. Phenothiazine and phenoxazine skeletons are deemed to be attractive frameworks for materials with redox-responsive functions, owing to these desirable properties. ${ }^{3}$ Incorporation of phenothiazine or phenoxazine skeletons in condensed polycyclic systems may produce compounds that exhibit excellent stimulus-responsive properties like PTZ and PXZ. The isolation and investigation of radical cations are essential to determine the differences in structural and electronic properties between neutral and oxidized states. However, only a few studies have reported on the isolation and structural elucidation of cationic species of condensed polycyclic compounds using phenothiazine or phenoxazine skeletons. In a recent work, we described the redox-induced planarization of polycyclic aromatic compounds using the phenoxazine substructure, trioxytriphenylamine TOT, and its application in the development of redox-responsive magnetic materials (Figure 1). ${ }^{3 c, d, 4}$

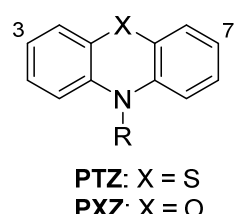<smiles>c1cc2c3c(c1)Oc1cccc4c1N3c1c(cccc1O4)O2</smiles>

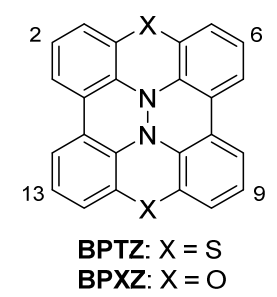

Figure 1. Chemical structure of electron donors with phenothiazine or phenoxazine substructures.

In the course of our attempts to explore condensed polycyclic aromatic systems with redox-induced planarization, we have focused on the condensed phenothiazine dimer BPTZ, previously reported by Higashibayashi et al. ${ }^{5}$ BPTZ has a characteristic double butterfly structure, but the molecular structure of the radical cation has not been described yet. Theoretical studies using density functional theory (DFT) calculations were carried out, suggesting that BPTZ $^{++}$has a non-planar structure, owing to the long C-S bond lengths. Shortening the carbon-heteroatom bond 
length by replacing sulfur with oxygen atoms in BPTZ is a reasonable strategy to achieve non-planar to planar transformation upon oxidation. This approach is further supported by theorical studies. Herein, we synthesized the condensed the phenoxazine dimer BPXZ and successfully isolated its radical cation. The molecular structures of BPXZ and $\mathbf{B P X Z}^{+}$were investigated by X-ray crystallographic analysis.

Scheme 1. Synthesis of BPXZ.<smiles>COc1cccc(Br)c1Nc1c(F)cccc1Br</smiles><smiles>CC(=O)c1ccc(F)c(Nc2c(O)cccc2Br)c1Nc1c(O)cccc1Br</smiles>

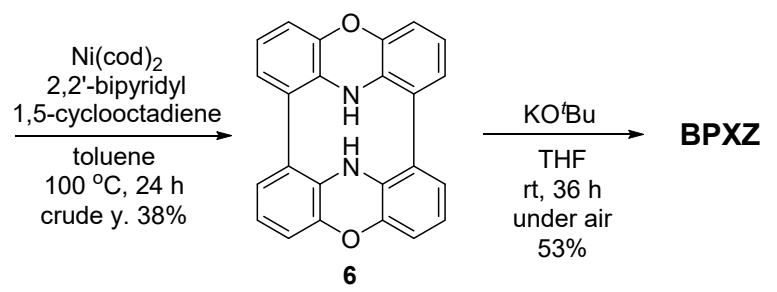

Scheme 1 illustrates the synthetic method to fabricate BPXZ. First, 3-bromo-2-iodoanisole (1) was synthesized according to a previously reported method. ${ }^{6}$ Following this, diarylamine $\mathbf{3}$ was obtained by the Buchwald-Hartwig cross-coupling reaction of $\mathbf{1}$ and 2-bromo-6-iodoaniline (2). ${ }^{7}$ Deprotection of the hydroxy group in $\mathbf{3}$ was carried out using $\mathrm{BBr}_{3}$, followed by the intramolecular aromatic nucleophilic substitution in the presence of $\mathrm{K}_{2} \mathrm{CO}_{3}$, thereafter producing 1,9-dibromo-10H-phenoxazine (5). ${ }^{4}$ The conversion of 5 into BPXZ was realized by slightly modifying the dimerization protocol developed by Higashibayashi as follows: ${ }^{5}$ phenoxazine dimer 6 was fabricated by the nickel-mediated homo-coupling reaction of 5 . N-N bond formation was achieved by the aerobic oxidation of $\mathbf{5}$ under alkaline conditions, generating BPXZ as a red solid in 53\% yield. Although BPXZ has a low solubility in common organic solvents, the structure of BPXZ was undoubtedly identified by MS, NMR, and X-ray structural and elemental analysis. BPXZ is stable under aerated conditions at room temperature in solid and solution states (Figure S1).

Single crystals of BPXZ suitable for X-ray crystallographic analysis were grown via the slow evaporation of THF from the solution. Four crystallographically independent molecules of BPXZ with almost identical structures in a unit cell were identified (Figure S3); ${ }^{8}$ Figures 2a-c displays one of the identified BPXZ structures. As expected, BPXZ adopts a non-planar structure, where each phenoxazine unit has flat butterfly shape with a dihedral angle of $167^{\circ}-169^{\circ}$ between the two benzene rings, bent in the opposite direction. Two oxygen atoms are closer to the mean square plane formed by carbon atoms, and two nitrogen atoms are positions above and below the mean square plane. The deviations out of the plane were $0.25-0.29 \AA$ and $0.36-0.37 \AA$ for oxygen and nitrogen atoms, respectively (Figure $2 \mathrm{~b}, \mathrm{c}$ ). The sum of $\mathrm{C}-\mathrm{N}-\mathrm{C}$ bond angles around each nitrogen atom is $336-337^{\circ}$, suggesting that the nitrogen atoms have significant $\mathrm{sp}^{3}$ hybridization. The $\mathrm{N}-\mathrm{N}$ bond lengths were $1.48-1.49 \AA$ which are longer than those reported for tetraphenylhydrazine derivatives $(1.38-1.43 \AA) .5 \mathrm{a}$ This can be attributed to the electronic repulsion between lone pair electrons of the nitrogen atoms. Moreover, $\mathrm{N}-\mathrm{N}$ bond elongation was reported for BPTZ and its derivatives (1.44-1.50 ̊̊). ${ }^{5}$ BPXZ molecules are arranged in 1-D slip-stacked columns along the $b$-axis with close contacts between the carbon atoms ( $3.38 \AA)$ and the carbon and oxygen atoms (3.21 $\AA$ ) (Figure 3a). These distances are slightly shorter than the sum of the van der Waals radii (C-C: $3.40 \AA$ and C-0: $3.22 \AA$ ). ${ }^{9}$ (a)

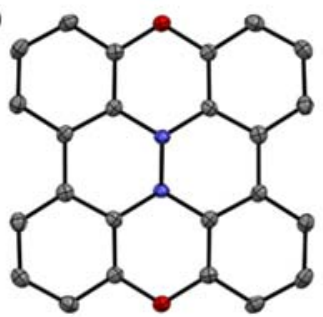

(d)

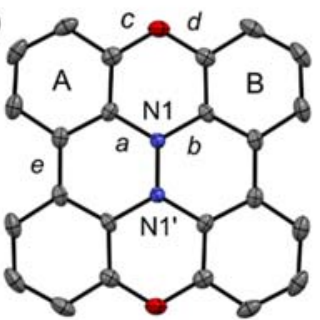

(b)

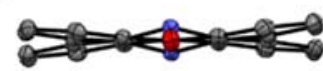

(c)

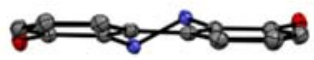

(e)

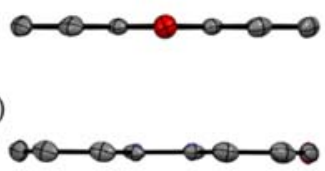

Figure 2. ORTEP views of BPXZ (upper) and $\mathbf{B P X Z}^{\cdot+\bullet \mathrm{SbF}_{6}}$ (lower) (50\% probabilities): Top (a) and (d) and side views (b), (c), (e) and (f). Hydrogen atoms and counter anions were omitted for clarity. Grey, blue, and red ellipsoids represent carbon, nitrogen, and oxygen atoms, respectively.

Table 1. The selected bond lengths ${ }^{\mathrm{a}}$ and the bond and dihedral angles of BPXZ and $\mathbf{B P X Z}{ }^{\cdot+}$.

\begin{tabular}{ccc}
\hline bonds and angles & $\mathbf{B P X Z}^{\mathrm{b}}$ & $\mathbf{B P X Z}^{{ }^{+} \cdot \mathbf{S b F}_{6}}{ }^{-}$ \\
$\mathrm{N}-\mathrm{N}$ & 1.49 & 1.37 \\
$a(\mathrm{C}-\mathrm{N})$ & 1.43 & 1.41 \\
$b(\mathrm{C}-\mathrm{N})$ & 1.43 & 1.40 \\
$c(\mathrm{C}-\mathrm{O})$ & 1.38 & 1.37 \\
$d(\mathrm{C}-\mathrm{O})$ & 1.38 & 1.37 \\
$e(\mathrm{C}-\mathrm{C})$ & 1.48 & 1.46 \\
Sum of bond angles & $336-337^{\circ}$ & $360^{\circ}$ \\
around the nitrogen atom & & \\
Dihedral angles between & $167-169^{\circ}$ & $180^{\circ}$ \\
A and B rings & &
\end{tabular}

aIn $\AA$ unit. bThe assignment of bond names are shown in Figure 2. cObtained from one of the independent molecules. 
(a)

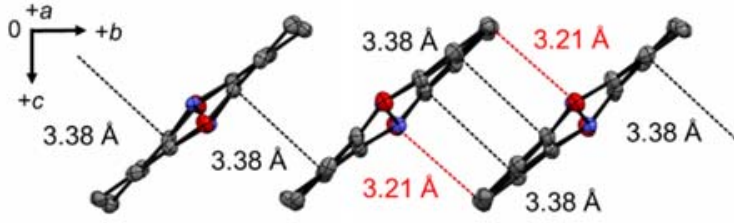

(b)

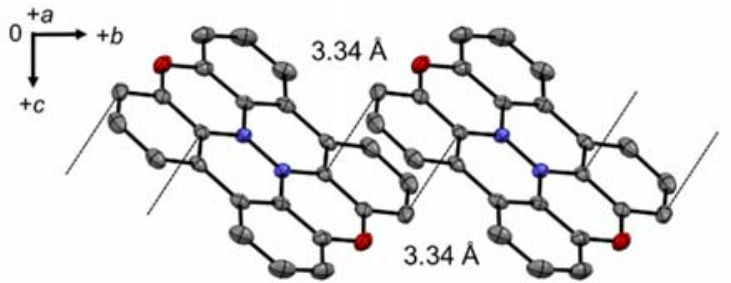

Figure 3. ORTEP views of crystal packing motifs of BPXZ (a) and $\mathbf{B P X Z}^{++\bullet \mathrm{SbF}_{6}}{ }^{-}$(b) (50\% probabilities) Hydrogen atoms and counter anions were omitted for clarity. Grey, blue, and red ellipsoids represent carbon, nitrogen, and oxygen atoms, respectively. Dash lines show short C-C (black) and C-O contacts (red).

To evaluate the stability of the radical cation BPXZ ${ }^{+}$, cyclic voltammograms of BPXZ were measured in degassed THF in the presence of $1.0 \mathrm{M}$ tetra- $n$-butylammonium perchlorate as a supporting electrolyte (Figure 4). A reversible redox wave was observed at $E_{1 / 2^{0 /+1}}=-0.20 \mathrm{~V}$ vs Fc $/ \mathrm{Fc}^{+}$because of the formation of $\mathbf{B P X Z}{ }^{\cdot+}$, indicating that $\mathbf{B P X Z} \mathbf{P}^{+}$is stable under these conditions. Neither the second oxidation nor the reduction wave was observed under the conditions of CV measurement. The first oxidation potential of BPXZ is slightly lower than that reported for BPTZ $\left(E_{1 / 2^{0 /+1}}=-0.12\right.$ $V) .{ }^{5 \mathrm{~b}}$ The low oxidation potential alludes to the strong electron-donating ability of BPXZ.

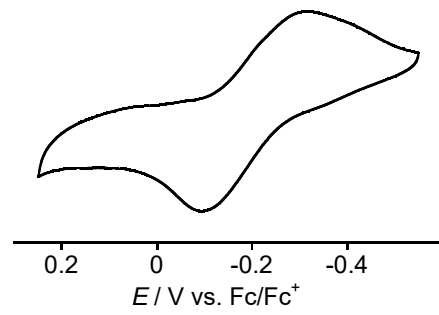

Figure 4. Cyclic voltammogram of BPXZ in THF in the presence of $0.1 \mathrm{M} n \mathrm{Bu}_{4} \mathrm{NClO}_{4} ; \mathrm{V} \mathrm{vs} \mathrm{Fc} / \mathrm{FC}^{+}=0 \mathrm{~V}(+0.55 \mathrm{~V}$ vs SCE$)$, working electrode: glassy carbon, counter electrode: platinum, reference electrode: SCE, scan rate $100 \mathrm{mV} / \mathrm{s}$.

Chemical oxidation of $\mathbf{B P X Z}$ was carried out in $\mathrm{CH}_{2} \mathrm{Cl}_{2}$ at room temperature using tris(4-bromophenyl)aminium hexafluoroantimonate as the oxidant. The desired radical cation salt $\mathbf{B P X Z}^{+} \cdot \mathrm{SbF}_{6}{ }^{-}$was obtained as a blue solid in $83 \%$ yield and its purification was achieved via the reprecipitation of the $\mathrm{CH}_{3} \mathrm{CN}$ solution using diethyl ether. The structure and properties of $\mathbf{B P X Z} \cdot{ }^{+} \cdot \mathrm{SbF}_{6}-$ were characterized by MS, EPR (electron paramagnetic resonance), X-ray crystallography and elemental analysis. The UV-vis-near infrared (NIR) spectrum of $\mathbf{B P X Z}^{\cdot+} \cdot \mathrm{SbF}_{6}{ }^{-}$in $\mathrm{CH}_{3} \mathrm{CN}$ (Figure 5) exhibited characteristic bands with absorption maxima at 633 and $693 \mathrm{~nm}$. Time-dependent DFT calculations
(UB3LYP/6-31G**//UB3LYP/6-31G**) suggested that the low-energy absorption band originates from the HOMO-1HOMO (SOMO) transition $(f=0.0658$ ) (Figure S2, Table S3). Moreover, $\mathbf{B P X Z}^{\cdot+} \cdot \mathrm{SbF}_{6}{ }^{-}$in $\mathrm{CH}_{3} \mathrm{CN}$ exhibited negligible spectral change after $24 \mathrm{~h}$ under aerated conditions at room temperature (Figure S2), indicating the highly stable nature of $\mathbf{B P X Z}^{*+}$ under these conditions.

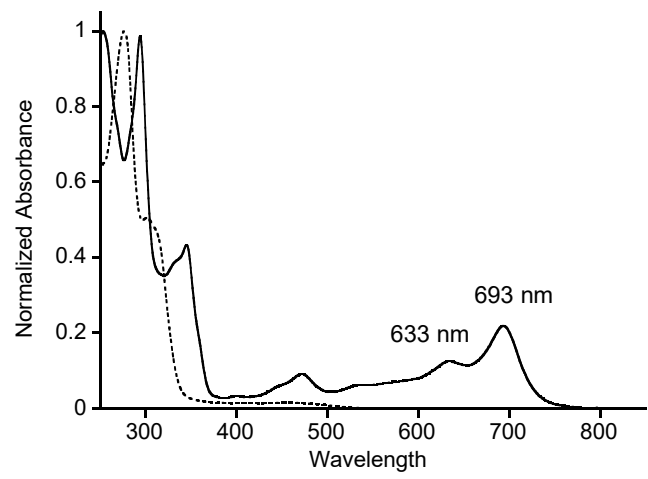

Figure 5. UV-vis-NIR absorption spectra of BPXZ in THF (dashed line) and $\mathbf{B P X Z}^{+\cdot} \cdot \mathrm{SbF}_{6}$ (solid line) in $\mathrm{CH}_{3} \mathrm{CN}$. (a)

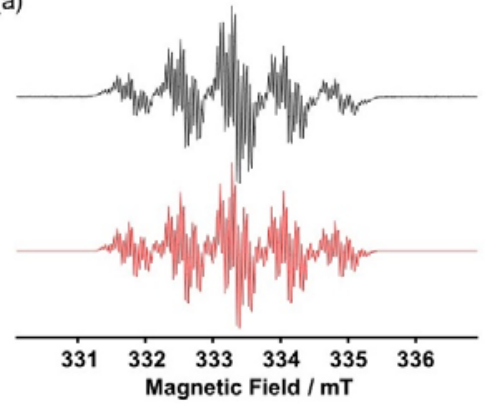

(b)

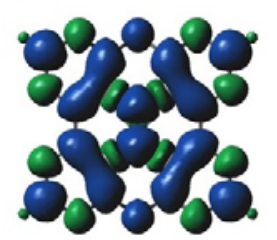

Figure 6. EPR spectra of BPXZ •*; observed in degassed $\mathrm{CH}_{3} \mathrm{CN}$ at room temperature, $9.346338 \mathrm{GHz}, g=2.0033$ (upper, black line) and simulated (lower, red line). Parameters for the simulation: $\left|a\left({ }^{14} \mathrm{~N}\right)\right|=0.761 \mathrm{mT},\left|a\left({ }^{1} \mathrm{H}\right)\right|=0.175 \mathrm{mT}, 0.0489 \mathrm{mT}$, $0.0366 \mathrm{mT}$. (b) Spin density map of $\mathbf{B P X Z}^{+}$calculated at the DFT UB3LYP/6-31G** level (isovalue $=0.0004$, blue positive spin, green: negative spin).

To gain further inside into the electronic structure, the EPR spectrum of $\mathbf{B P X Z}^{\cdot+\bullet \mathrm{SbF}_{6}-}$ was obtained in degassed $\mathrm{CH}_{3} \mathrm{CN}$ at room temperature. Multiple split lines with $g=$ 2.0033 were observed (Figure 6). This spectral lines were successfully reproduced using spectral simulation with the following hyperfine coupling constants: ${ }^{14} \mathrm{~N}$ nuclei $(I=1)$, $a\left({ }^{14} \mathrm{~N}\right)=7.17 \times 10^{-4} \mathrm{~cm}^{-1}(0.761 \mathrm{mT})$; the three sets of ${ }^{1} \mathrm{H} \mathrm{nu}-$ clei $(I=1 / 2):\left|a\left({ }^{1} \mathrm{H}\right)\right|(\times 4)=1.65 \times 10^{-4} \mathrm{~cm}^{-1}(0.175 \mathrm{mT})$, $\left|a\left({ }^{1} \mathrm{H}\right)\right|(\times 4)=4.61 \times 10^{-5} \mathrm{~cm}^{-1}(0.0489 \mathrm{mT})$, and $\left|a\left({ }^{1} \mathrm{H}\right)\right|(\times 4)$ $=3.45 \times 10^{-5} \mathrm{~cm}^{-1}(0.0366 \mathrm{mT})$. The largest coupling constant of ${ }^{1} \mathrm{H}$ nuclei was reasonably assigned, with the aid of the theoretical calculations, to the four equivalent ${ }^{1} \mathrm{H}$ nuclei at the 2, 6, 9, and 13-positions of BPXZ (Figure 6b), suggesting that the incorporation of a radical unit at these positions leads to a stronger spin-spin coupling. These results indicate that the electron spin of $\mathbf{B P X Z}^{\cdot+}$ is delocalized over the 
entire $\pi$-conjugated system. Low temperature EPR of BPXZ $^{*+}$ in solid sample was measured at $110 \mathrm{~K}$. Unfortunately, no notable intermolecular magnetic interaction is observed (Figure S6).

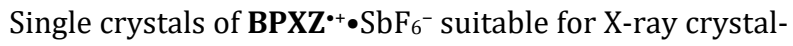
lographic analysis were obtained via slow diffusion of ether

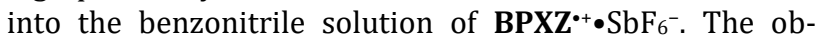
served molecular structure of $\mathbf{B P X Z}{ }^{-+}$is shown in Figure $2 \mathrm{~d}-$ f. BPXZ ${ }^{\cdot+}$ presents a planar structure with a dihedral angle of $180^{\circ}$ between rings $A$ and $B$, and an inversion center located in the middle of the N1-N1' bond. The summation of $\mathrm{C}-\mathrm{N}-\mathrm{C}$ bond angles around the nitrogen atom increased to $360^{\circ}$ upon oxidation, indicating that the nitrogen atoms have an $\mathrm{sp}^{2}$ hybridization instead of $\mathrm{sp}^{3}$. The N1-N1' bond-length was significantly shortened from $1.49 \AA$ to $1.37 \AA ̊$ upon oxidation because of the reduction in electron repulsion between the unshared electrons of the nitrogen atoms (Table 1). These results confirm that, upon oxidation, the BPXZ unit structure was converted from non-planar double butterfly to planar. In addition, the bond length analysis showed that all $\mathrm{C}-\mathrm{O}, \mathrm{C}-\mathrm{N}$, and $\mathrm{C}-\mathrm{C}$ bonds bridging the two phenoxazine units are shorter than the corresponding bonds in neutral BPXZ, indicating $\pi$-electron delocalization over the entire molecule. HOMA (harmonic oscillator model of aromaticity) indexes were evaluated for heteroatom-containing rings in BPXZ (0.0405) and BPXZ ${ }^{-+}(0.270){ }^{10}$ These HOMA indexes indicated that the aromatic character of the heteroatomcontaining ring is enhanced upon oxidation. BPXZ ${ }^{++}$molecules formed 1-D slipped $\pi$-stacks along the $b$-axis with interplanar distances of $3.34 \AA$. This distance is shorter than the sum of van der Waals radii of carbon atoms (3.40 $\AA)$ (Figure $3 \mathrm{~b}$ ). The stacking columns were aligned along the $c$ axis with a short contact between the carbon and oxygen atoms (3.28 ̊) (Figure S5).

In conclusion, a condensed phenoxazine dimer was synthesized and oxidized to produce its stable radical cation salt, which was isolated as a blue solid. Electrochemical measurements revealed that the condensed dimer is a strong electron donor. Crystal structures of the neutral species and radical cation salt were elucidated using X-ray crystallographic analysis. The neutral species adopts a characteristic double-butterfly structure, while the radical cation exhibited a planar structure. These results suggest that the condensed dimer is attractive for application as building blocks in redox-responsive magnetic materials.

\section{ASSOCIATED CONTENT}

\section{Supporting Information}

The Supporting Information is available free of charge on the ACS Publications website.

Detailed experimental procedures, characterization data, X-ray data with packing structure, copies of ${ }^{1} \mathrm{H}$ and ${ }^{13} \mathrm{C}$ NMR spectra of products, and DFT-calculations.

\section{Accession Codes}

CCDC 2014695 (BPXZ) and 2014696 (BPXZ ${ }^{\bullet+S}$ bF $_{6}{ }^{-}$) contains the supplementary crystallographic data for this paper. These data can be obtained free of charge via www.ccdc.cam.ac.uk/data_request/cif, or by emailing data_request@ccdc.cam.ac.uk, or by contacting The Cambridge Crystallographic Data Centre, 12 Union Road, Cambridge CB2 1EZ, UK; fax: +44 1223336033 .

\section{AUTHOR INFORMATION}

\section{Corresponding Author}

*E-mail: suzuki-s@chem.es.osaka-u.ac.jp

*E-mail: kozaki@sci.osaka-cu.ac.jp

\section{Author Contributions}

All authors have given approval to the final version of the manuscript.

Notes

'deceased

\section{ACKNOWLEDGMENT}

This work was partially supported by Grant-in-Aid for Scientific Research (JSPS KAKENHI \#Grant Numbers, JP15H00956 (K.O.) JP17K05790 (M.K. and K.O.) and JP26102005 (S.S), JP17K05783 (S.S)).

\section{REFERENCES}

(1) (a) Organic Redox Systems (Ed.: T. Nishinaga), John Wiley \& Sons, Inc., New Jersey, 2016. (b) Hirai, M.; Tanaka, N.; Sakai, M.; Yamaguchi, S. Chem. Rev. 2019, 119, 14, 8291-8331. (c) Sucunza, D.; Cuadro, A. M.; Alvarez-Builla, J.; Vaquero, J. J. J. Org. Chem. 2016, 81 , 10126-10135. (d) Abe, M. Chem. Rev. 2013, 113, 7011-7088.

(2) (a) Oka, H. Org. Lett. 2010, 12, 448-451. (b) Tahara, T.; Suzuki, S.; Kozaki, M.; Shiomi, D.; Suigisaki, K.; Sato, K.; Takeji, T.; Miyake, Y.; Hosokoshi, Y.; Noijiri, H.; Okada, K. Eur. J. Chem. 2019 25, 7201-7209. (c) Suzuki, S.; Maya, R.; Uchida, Y.; Naota, T. ACS Omega, 2019, 4, 10031-10035.

(3) (a) Satoh, Y.; Catti, L.; Akita, M.; Yoshizawa, M. J. Am. Chem. Soc. 2019, 141, 12268-12273. (b) Suzuki, S.; Nagata, A.; Kuratsu, M; Kozaki, M.; Tanaka, R.; Shiomi, D.; Sugisaki, K.; Toyota, K.; Sato, K.; Takui, T.; Okada, K. Angew. Chem. Int. Ed. 2012, 51, 3193-3197. (c) Kuratsu, M.; Suzuki, S.; Kozaki, M.; Shiomi, D.; Sato, K.; Takui, T.; Kanzawa, T.; Hosokoshi, Y.; X.-Z. Lan, Y. Miyazaki, A. Inaba, K. Okada, K. Chem. Asian J. 2012, 7, 1604-1609.

(4) Kuratsu, M.; Kozaki, M.; Okada, K. Angew. Chem. Int. Ed. 2005, 44, 4056-4058.

(5) (a) Yamamoto, K.; Higashibayashi, S. Chem. Eur. J. 2016, 22, 663-671. (b) Shindo, Y.; Nomura, S.; Saikawa, Y.; Nakata, M. Tanaka, K.; Hanaya, K.; Sugai, T.; Higashibayashi, S. Asian J. Org. Chem. 2018, 7, 1797-1801.

(6) (a) Sanz, R.; Castroviejo, M. P.; Fernandez, Y.; Fananas, F. J. J. Org. Chem. 2005, 70, 6548-6551. (b) Sanz, R.; Castroviejo, M. P.; Guilarte, V.; Perez, A.; Fananas, F. J. J. Org. Chem. 2007, 72, 51135118.

(7) Wolfe, J. P.; Wagaw, S.; Marcoux, J.-F.; Buchwald, S. L. Acc. Chem. Res. 1998, 31, 805-818.

(8) One of four molecules has disorder in its structure.

(9) Bondi, A. J. Phys. Chem. 1964, 68, 441-451.

(10) Krygowski, T. M.; Szatylowicz, H.; Stasyuk, O. A.; Dominikowska, J.; Palusiak, M. Chem. Rev. 2014, 114, 6383-6422. 